\title{
Public Health Implications of Human Papillomavirus Oral Infection: A Brief Overview
}

\author{
Amit Chattopadhyay • Darien Weatherspoon • \\ Andres Pinto
}

Published online: 2 July 2014

(C) Springer International Publishing AG 2014

\begin{abstract}
Human papilloma virus (HPV) types 16 and 18 are associated with cancers of the cervix and oropharyngeal region. Two kinds of HPV vaccines (bivalent and quadravalent) available in the market for cervical cancer prevention are safe and efficacious. Because the same types of HPV that cause cervical cancer are also associated with oropharyngeal cancers, it is thought that these vaccines may also be effective in prevention of these cancers. A call for extending the indication for these vaccines to include oropharyngeal cancer prevention has been made. Oral health professionals, who are in the forefront of visualizing the oral and oropharyngeal areas, are poised to detect abnormal findings in these regions and should consider discussing HPV vaccines with their patients. This brief review discusses the role of HPV in oral lesions and oropharyngeal cancers and the public health implications of this infection.
\end{abstract}

Keywords HPV · Oral cancer · Oral diseases · Oropharyngeal cancer $\cdot$ Vaccine $\cdot$ Prevention

\section{Introduction}

Human Papillomavirus (HPV) is a small, circular double stranded DNA virus with a large number of genotypes, having some 8,000 base pairs in its genome. Several types of HPV

A. Chattopadhyay $(\triangle) \cdot$ D. Weatherspoon

SCASI Research, Gaithersburg, MD, USA

e-mail: ac@sharmilachatterjee.com

\section{A. Pinto}

Department of Oral and Maxillofacial Medicine and Diagnostic Sciences, School of Dental Medicine, Case Western Reserve

University Dental School, Cleveland, OH, USA have high oncogenic potential. These viruses are well adapted to their host and cleverly sequestered away from immune responses. In general, HPV is associated to cervical, anal, vaginal, vulvar, penile, oropharyngeal, and select skin cancers [1]. The largest impact of HPV on human health is due to its causal association with cervical cancer, the second most common cancer among women. It is estimated by the World Health Organization that world-wide, approximately 2,337 million women age 15 and older are at risk of developing cervical cancer. Of these, $77.5 \%$ live in developing regions. HPV types 16 and 18 are considered to be high risk viruses and are responsible for about $70 \%$ of all cervical cancer cases worldwide $[2 \bullet$.

This brief review discusses the role of HPV in oral health; strategies for HPV infection prevention; the role of HPV vaccines in cervical cancer prevention; their costs and current HPV vaccine policy; and oral health professionals' preparedness to discuss HPV vaccination with patients.

\section{HPV in the Oral Cavity}

Human Papilloma Virus is not commonly isolated from the oral cavity. HPV oral carriage varies between $1.9 \%$ and $10 \%$ in children, adolescents and adults [3-5]. It has been suggested that distribution of HPV in the oral cavity may be impacted by the topography of the mucosa, and the virus is predominantly present in the vermilion border, labial commissures and hard palate [6]. A study assessing the 2009-2010 National Health and Nutrition Examination Survey data reported an association of higher unadjusted and adjusted prevalence of oral HPV infection with four measures of oral health: self-rated poor oral health; possibility of gingival disease; reported use of mouthwash to treat dental problems in the past week; and a higher number of lost teeth $[7 \bullet \bullet]$. A recent 
systematic review described the prevalence of oral HPV infection to range between $2.6 \%$ to $50 \%$ in different studies [8]. Overall, of all the known types of HPV, about 25 types have been detected in oral cavity lesions [9॰].

\section{HPV and Oral Diseases}

HPV is associated with several oral diseases such as: oral warts (verruca vulgaris, squamous cell papilloma, and condyloma acuminatum); focal epithelial hyperplasia; oral leukoplakia with dysplastic change $[9 \cdot, 10]$; lichen planus; leukoplakia; erythroplakia; verrucous carcinoma; oral cancer; and oropharyngeal cancer [11].

\section{HPV, Oropharyngeal and Oral Cavity Cancer}

The role of HPV in oral and oropharyngeal cancers has been explored for more than a decade $[12,13]$. It is common knowledge that oral cancers are related to smoking and alcohol. Nevertheless, several studies illustrate a strong association between oropharyngeal cancers and HPV infection, irrespective of tobacco and alcohol use $[3,14,15]$. An ecological association between oral cancer incidence and HPV-related oropharyngeal cancer was observed between 1983 and 2002 in a study that compared incidence trends for oropharyngeal cancers, oral cavity cancers, and lung cancers. This study compared the role of HPV vis-à-vis smoking on incidence trends using data from the Cancer Incidence in Five Continents database Volumes VI to IX (years 1983 to 2002) [16••]. Oral transmission of HPV infection remains a major risk factor for HPV-associated oral and oropharyngeal cancers [17]. Interestingly, HPV-positive squamous cell carcinomas of the head and neck may show better prognosis and response to treatment compared to their HPV-negative counter parts [18-20]. HPV-16 is the most often implicated HPV type in oral and oropharyngeal cancer, and has been detected in $82 \%$ oropharyngeal cancers, compared to approximtely $10 \%$ of oral cavity cancers $[21 \bullet \bullet, 22]$.

The importance of HPV infection to overall health is underscored by recent evidence supporting a grossly underestimated prevalence of the infection in the USA. Doubts were raised regarding the precision of recorded death rates (and cause of death) from routine mortality statistics in the USA, which could impact HPV-related cancer mortality statistics. One study reported that routine mortality statistics (based on underlying cause of death), underestimated deaths due to oral cavity and oropharyngeal cancers among those below 65 years of age by about $22 \%-35 \%$; and underestimated the underlying cause of death secondary to tonsillar cancer (associated with HPV) by approximately $70 \%-80 \%[23]$.

\section{HPV Infection Transmission and Prevention}

\section{Transmission}

Transmission of HPV can be done through sexual contact, vertical transmission from infected mother to child during pregnancy, and mouth-to-mouth [24]. Evidence for oralgenital transmission of HPV is supported by a study examining HPV type concordance between couples. Oral-oral concordance of HPV type between couples was low, but concordances of oral-genital and genitalgenital HPV types were higher. HPV type concordance of male oral HPV infection with their partners' vaginal HPV infection was also reported to be high [25••]. Other known risk factors for oral and oropharyngeal cancers such as smoking and alcohol may increase the risk of transformation following a high-risk HPV infection [26].

\section{Prevention}

Interruption of transmission routes is a key factor that drives prevention of infection. In the case of HPV, prevention of infection includes the practice of safe sex. Prophylactic vaccine use is becoming an important tool to address cervical cancer prevention.

\section{Vaccination}

Current vaccines have the potential to reduce the incidence of cervical and other anogenital cancers [2•]. The US Food and Drug Administration has allowed license for two kinds of HPV vaccines that are effective against HPV types 6, 11, 16, 18. Of these vaccines, one is a bivalent vaccine (marketed as Cervarix ${ }^{\circledR}$ ), indicated for the prevention of cervical cancer and precancerous lesions in women. The second is a quadravalent vaccine (marketed as Gardasi ${ }^{\circledR} 1 /$ Silgard $^{\circledR}$ ), indicated for: the prevention of cervical cancer; precancerous lesions and genital warts associated with HPV in females; prevention of vaginal and vulvar cancer in females; prevention of genital warts in males; and prevention of anal cancer and precancerous lesions in both males and females [27, 28••]. Both vaccines are given as intramuscular injections in three doses over a six month period [29].

Policy and Costs

The American College of Obstetricians and Gynecologists endorsed the HPV vaccine recommendations of the Advisory Committee on Immunization Practices from the Centers for Disease Control and Prevention. This recommendation states that HPV vaccination should be routinely targeted to females 
and males aged 11 or 12 as part of the adolescent immunization platform, to help reduce the incidence of anogenital cancers and genital warts associated with HPV infection [30]. The quadravalent HPV vaccine is approved for use in males and females, whereas the bivalent HPV vaccine is approved for use only in females. For those not vaccinated at the target age, catch-up vaccination is recommended up to age 26 years.

It is estimated that the cost of each vaccination period is of about US\$360 [31]. A recent South African study assessed whether a cervical cancer prevention program incorporating an HPV vaccine was more cost-effective than the current South African strategy of screening for cervical cancer alone. The results revealed that to make the vaccine plus cancer screening program more cost effective than the current screening only program, the vaccine price would need to be reduced by more than $60 \%$ of the current prices [32]. A comprehensive review of eight published economic evaluations of universal HPV vaccination discussed the point that studies of the cost-effectiveness of universal HPV vaccination capture only a fraction of the values generated, and most studies focus on the impact on health and health system cost. Most studies tended to fully or partially exclude important values such as impact on productivity, patient time and costs, costs of caregivers and family costs, and broader social values such as the right to access treatment [33].

In the USA, legal and policy statements regarding HPV vaccination vary from State to State. States with HPV vaccination laws and policies are enumerated and briefly reviewed by Osazuwa-Peters [34••]. At this time, HPV vaccines are not recommended for prevention of cancers of the oral cavity or oropharyngeal region, although they are highly effective against the HPV strains that are commonly found in the oropharynx. It has been suggested that "with emerging evidence of the efficacy of the use of the HPV vaccine in preventing oral-HPV, more focus should be put on extending $\mathrm{HPV}$ vaccine to present oral HPV infection and oropharyngeal cancers" [34・•].

\section{Impact of HPV Vaccine on Oral and Oropharyngeal Cancer}

The effectiveness of these vaccines in the prevention of HPVpositive oropharyngeal cancer is unknown [21・•]. Because HPV-16 has been identified in approximately $90 \%$ of HPVpositive oropharyngeal cancers; it is assumed that current HPV vaccines may hold potential for preventing these cancers. At present, no clinical trials or other studies have demonstrated an oropharyngeal cancer prevention effect of these vaccines. A double-blind controlled trial investigating vaccine efficacy of the bivalent HPV 16/18 vaccine found that oral
HPV prevalence four years after vaccination was substantially reduced in the vaccine group compared to controls [35], providing insight into presumed effects on oral HPV viremia.

\section{HPV Vaccine and the Oral Health Professional}

It is obvious that oral health care personnel should be knowledgeable not just about the role of HPV in carcinogenesis and the association of HPV with oropharyngeal cancers but also about the efficacy, effectiveness, and potential impact of HPV vaccines. The current readiness of oral health professionals to disseminate HPV related prevention and vaccine information is controversial. A recent study from Florida, USA, evaluated oral health providers' intention and capacity for engaging in primary and secondary prevention of HPV-related oral cancers. The authors reported that only a small proportion of the study sample responded to the survey questions and most participants $(97 \%)$ fell into the pre-contemplation and contemplation stages of readiness to discuss the HPV vaccine with patients [36••].

Oral health professionals should be prompt in referring patients with suggestive signs and symptoms for evaluation and thereby play an important role in increasing patients' knowledge about HPV and oropharyngeal cancers [21••], as well as improving the reach of HPV infection prevention methods, vaccination, and timely treatment.

\section{Conclusion}

Significant progress has been made on the knowledge of the role of HPV in oral diseases. Epidemiologic and molecular evidence points to HPV as a major etiologic factor in oropharyngeal cancers. This causal association has garnered strong attention because of the public health implications of preventive approaches and vaccination. While current evidence suggests that HPV-positive oral and oropharyngeal cancers may have a better prognosis and survival than HPV-negative oral and oropharyngeal cancers; more research will be necessary to confirm this finding and to demonstrate the underlying mechanism(s) responsible for these characteristics.

Perhaps the greatest public health implication of HPVassociated oral disease is the potential role that an HPV vaccine could play in its prevention. To date, evidence for the effectiveness of the HPV vaccine for prevention of oral cavity and oropharyngeal cancers has been promising; however, more research and clinical trials will be necessary before HPV vaccination can be recommended for the prevention of HPV-related oral disease. As the effectiveness of the HPV vaccine in preventing HPV-associated oral diseases becomes more clear, it could very well have a widespread impact on HPV vaccine costs and policy. Additionally, oral health 
professionals should keep up- to-date on current information on HPV-associated oral diseases, so that they are prepared to effectively communicate this information to their patients.

\section{Compliance with Ethics Guidelines}

Conflict of Interest Dr. Amit Chattopadhyay and Dr. Darien Weatherspoon each declare no potential conflicts of interest.

Dr. Andres Pinto is a section editor for Current Oral Health Reports.

Human and Animal Rights and Informed Consent This article does not contain any studies with human or animal subjects performed by any of the authors.

\section{References}

Papers of particular interest, published recently, have been highlighted as:

- Of importance

.• Of major importance

1. NCI. Human papillomavirus. URL; http://www.cancer.gov/Common/ PopUps/popDefinition.aspx? id $=$ CDR0000045714\&version= Patient\&language=English. Last accessed May 18, 2014.

2. WHO/ICO Information Centre on HPV and Cervical Cancer (HPV Information Centre). Human Papillomavirus and Related Cancers in World. Summary Report 2010. [Date accessed]. Available at www.who.int/hpvcentre. This covers details about worldwide distribution of HPV-related cancers.

3. D'Souza G, Agrawal Y, Halpern J, Bodison S, Gillison ML. Oral sexual behaviors associated with prevalent oral human papillomavirus infection. J Infect Dis. 2009;199(9):1263-9.

4. Smith EM, Swarnavel S, Ritchie JM, Wang D, Haugen TH, Turek LP. Prevalence of human papillomavirus in the oral cavity/ oropharynx in a large population of children and adolescents. Pediatr Infect Dis J. 2007;26(9):836-40.

5. Summersgill KF, Smith EM, Levy BT, Allen JM, Haugen TH, Turek LP. Human papillomavirus in the oral cavities of children and adolescents. Oral Surg Oral Med Oral Pathol Oral Radiol Endod. 2001;91(1):62-9.

6. Mravak-Stipetic M, Sabol I, Kranjcic J, Kneževic M, Grce M. Human papillomavirus in the lesions of the oral mucosa according to topography. PLoS One. 2013;8(7):e69736.

7.• Bui TC, Markham CM, Ross MW, Mullen PD. Examining the association between oral health and oral HPV infection. Cancer Prev Res (Phila). 2013;6(9):917-24. Nice overview of HPV and oral health implications.

8. Termine N, Giovannelli L, Matranga D, Caleca MP, Bellavia C, Perino A, et al. Oral human papillomavirus infection in women with cervical HPV infection: new data from an Italian cohort and a metanalysis of the literature. Oral Oncol. 2011;47(4):244-50.

9. Syrjänen S, Lodi G, von Bültzingslöwen I, Aliko A, Arduino P, Campisi G, et al. Human papillomaviruses in oral carcinoma and oral potentially malignant disorders: a systematic review. Oral Dis. 2011;17 Suppl 1:58-72. Important meta-analysis of oral cancer and HPV and potentially malignant lesions. This is a recent excellent and important systematic review about oral cancers and HPV.

10. Syrjänen KJ. Human papillomavirus (HPV) infections and their associations with squamous cell neoplasia. Arch Geschwulstforsch. 1987;57(6):417-44.
11. Grce M, Mravak-Stipetic M. Human papillomavirus-associated diseases. Clin Dermatol. 2014;32(2):253-8.

12. Sturgis EM, Cinciripini PM. Trends in head and neck cancer incidence in relation to smoking prevalence: an emerging epidemic of human papillomavirus-associated cancers? Cancer. 2007;110(7): 1429-35.

13. Chaturvedi AK, Engels EA, Anderson WF, Gillison ML. Incidence trends for human papillomavirus-related and -unrelated oral squamous cell carcinomas in the United States. J Clin Oncol Off J Am Soc Clin Oncol. 2008;26(4):612-9.

14. Lambert R, Sauvaget C, de Camargo Cancela M, Sankaranarayanan R. Epidemiology of cancer from the oral cavity and oropharynx. Eur $\mathrm{J}$ Gastroenterol Hepatol. 2011;23(8):633-41.

15. Gillison ML, Koch WM, Capone RB, Spafford M, Westra WH, Wu L, et al. Evidence for a causal association between human papillomavirus and a subset of head and neck cancers. J Natl Cancer Inst. 2000;92(9):709-20.

16.• Chaturvedi AK, Anderson WF, Lortet-Tieulent J, Curado MP, Ferlay J, Franceschi S, et al. Worldwide trends in incidence rates for oral cavity and oropharyngeal cancers. J Clin Oncol. 2013;31(36):4550-9. Excellent source for updated epidemiologic data on oral and oropharyngeal cancer.

17. Gillison ML. Human papillomavirus-related diseases: oropharynx cancers and potential implications for adolescent HPV vaccination. J Adolesc Health. 2008;43(4 Suppl):S52-60.

18. Kaka AS, Kumar B, Kumar P, Wakely Jr PE, Kirsch CM, Old $\mathrm{MO}$, et al. Highly aggressive human papillomavirusrelated oropharyngeal cancer: clinical, radiologic, and pathologic characteristics. Oral Surg Oral Med Oral Pathol Oral Radiol. 2013;116(3):327-35.

19. Ragin CC, Taioli E. Survival of squamous cell carcinoma of the head and neck in relation to human papillomavirus infection: review and meta-analysis. Int J Cancer. 2007;121(8): 1813-20.

20. Fakhry C, Westra WH, Li S, Cmelak A, Ridge JA, Pinto H, et al. Improved survival of patients with human papillomavirus-positive head and neck squamous cell carcinoma in a prospective clinical trial. J Natl Cancer Inst. 2008;100(4):261-9.

21.• Cleveland JL, Junger ML, Saraiya M, Markowitz LE, Dunne EF, Epstein JB. The connection between human papillomavirus and oropharyngeal squamous cell carcinomas in the United States: implications for dentistry. J Am Dent Assoc. 2011;142(8):915-24. Population based analysis if HPV and oropharyngeal cancer.

22. Kreimer AR, Clifford GM, Boyle P, Franceschi S. Human papillomavirus types in head and neck squamous cell carcinomas worldwide: a systematic review. Cancer Epidemiol Biomarkers Prev. 2005;14(2):467-75.

23. Polednak AP. Inaccuracies in oral cavity-pharynx cancer coded as the underlying cause of death on U.S. death certificates, and trends in mortality rates (1999-2010). Oral Oncol. 2014. doi: 10.1016/j. oraloncology.2014.04.012.

24. Rautava J, Syrjanen S. Human papillomavirus infections in the oral mucosa. J Am Dent Assoc. 2011;142:905-14.

25.• Vogt SL, Gravitt PE, Martinson NA, Hoffmann J, D'Souza G. Concordant oral-genital HPV infection in South Africa couples: evidence for transmission. Front Oncol. 2013;3:303. This study offers evidence behind oral/sexual HPV transmission.

26. Schiffman M, Castle PE, Jeronimo J, Rodriguez AC, Wacholder S. Human papillomavirus and cervical cancer. Lancet. 2007;370(9590):890-907.

27. Dochez C, Bogers JJ, Verhelst R, Rees H. HPV vaccines to prevent cervical cancer and genital warts: an update. Vaccine. 2014;32(14): 1595-601.

28.• Harper DM, Demars LR. Primary strategies for HPV infection and cervical cancer prevention. Clin Obstet Gynecol. 2014;57(2):256-78. This is an excellent review of HPV infection prevention strategies. 
29. World Health Organization. Human papillomavirus vaccines. WHO position paper: Weekly epidemiological record 2009. 2009;84(15):118-31.

30. Committee on Adolescent Health Care of the American College of Obstetricians and Gynecologists; Immunization Expert Work Group of the American College of Obstetricians and Gynecologists. Committee opinion no. 588: human papillomavirus vaccination. Obstet Gynecol. 2014;123(3):712-8.

31. Insinga RP, Dasbach EJ, Elbasha EH. Assessing the annual economic burden of preventing and treating anogenital human papillomavirus-related disease in the US: analytic framework and review of the literature. Pharmacoeconomics. 2005;23(11):110722.

32. Sinanovic E, Moodley J, Barone MA, Mall S, Cleary S, Harries J. The potential cost-effectiveness of adding a human papillomavirus vaccine to the cervical cancer screening programme in South Africa. Vaccine. 2009;27(44):6196-202.
33. Marsh K, Chapman R, Baggaley RF, Largeron N, Bresse X. Mind the gaps: what's missing from current economic evaluations of universal HPV vaccination? Vaccine. 2014. doi:10.1016/j.vaccine. 2014.05.007.

34.• Osazuwa-Peters N. Human papillomavirus (HPV), HPV-associated oropharyngeal cancer, and HPV vaccine in the United States-do we need a broader vaccine policy? Vaccine. 2013;31(47):5500-5. Public health implications of vaccination.

35. Herrero R, Quint W, Hildesheim A, Gonzalez P, Struijk L, Katki HA, et al. Reduced prevalence of oral human papillomavirus (HPV) 4 years after bivalent HPV vaccination in a randomized clinical trial in Costa Rica. PLoS One. 2013;8(7):e68329.

36.• Daley E, Dodd V, DeBate R, Vamos C, Wheldon C, Kline N, et al. Prevention of HPV-related oral cancer: assessing dentists' readiness. Public Health. 2014;128(3):231-82. This study reports the insufficient readiness of dentists to counsel patients about HPV infection/ risks. 